BMJ Open

Diabetes

Research

\& Care

\title{
Nicorandil attenuates high glucose- induced insulin resistance by suppressing oxidative stress-mediated ER stress PERK signaling pathway
}

\author{
Zhongwei Liu, ${ }^{1}$ Haitao Zhu, ${ }^{2}$ Chunhui He, ${ }^{3}$ Ting He, ${ }^{3}$ Shuo Pan, ${ }^{1}$ Na Zhao, \\ Ling Zhu, ${ }^{1}$ Gongchang Guan, ${ }^{1}$ Peng Liu, ${ }^{1}$ Yong Zhang, ${ }^{1}$ Junkui Wang (D) ${ }^{1}$
}

To cite: Liu Z, Zhu H, He C, et al. Nicorandil attenuates high glucose-induced insulin resistance by suppressing oxidative stress-mediated ER stress PERK signaling pathway. BMJ Open Diab Res Care 2021;9:e001884. doi:10.1136/ bmjdrc-2020-001884

- Supplemental material is published online only. To view, please visit the journal online (http://dx.doi.org/10.1136/ bmjdrc-2020-001884).

Received 3 September 2020 Revised 3 March 2021 Accepted 28 March 2021

Check for updates

C Author(s) (or their employer(s)) 2021. Re-use permitted under CC BY-NC. No commercial re-use. See rights and permissions. Published by BMJ.

${ }^{1}$ Department of Cardiology, Affiliated Shaanxi Provincial People's Hospital, Northwestern Polytechnical University, Xi'an, China

2Department of Pediatrics, Northwest Women's and Children's Hospital, Xi'an, China ${ }^{3}$ Department of Cardiology, Fuwai Hospital State Key Laboratory of Cardiovascular Disease, Beijing, China

Correspondence to Dr Junkui Wang; junkuiwang@yeah.net and Dr Yong Zhang;

zhangyong971292@163.com

\section{ABSTRACT}

Introduction Glucose-induced insulin resistance is a typical character of diabetes. Nicorandil is now widely used in ischemic heart disease. Nicorandil shows protective effects against oxidative and endoplasmic reticulum (ER) stress, which are involved in insulin resistance. Here, we investigated mechanisms of nicorandil's novel pharmacological activity on insulin resistance in diabetes. Research design and methods Nicorandil was administrated to streptozotocin-induced animals with diabetes and high glucose exposed skeletal muscle cells. Insulin resistance and glucose tolerance were evaluated. Molecular mechanisms concerning oxidative stress, ER stress signaling activation and glucose uptake were assessed.

Results Nicorandil attenuated high glucose-induced insulin resistance without affecting fasting blood glucose and glucose tolerance in whole body and skeletal muscle in rats with diabetes. Nicorandil treatment suppressed protein kinase $\mathrm{C} /$ nicotinamide adenine dinucleotide phosphate oxidases system activities by reducing cytoplasmic free calcium level in skeletal muscle cells exposed to high glucose. As a result, the oxidative stressmediated ER stress protein kinase RNA-like endoplasmic reticulum kinase (PERK)/eukaryotic initiation factor $2 \alpha /$ activating transcription factor 4/CEBP homologous protein/ tribbles homolog (TRB)3 signaling pathway activation was inhibited. Nicorandil downregulated expression of TRB3 and thus facilitated Akt phosphorylation in response to insulin stimulation, leading to glucose transporter4 plasma membrane translocation which promoted glucose uptake capability of skeletal muscle cells.

Conclusions By reducing cytoplasmic calcium, nicorandil alleviated high glucose-induced insulin resistance by inhibiting oxidative stress-mediated ER stress PERK pathway.

\section{INTRODUCTION}

The mortality and morbidity of diabetes have been increasing rapidly worldwide. Insulin resistance (IR), namely the resistance of tissue in response to insulin stimulation, is a characterized pathophysiological feature of diabetes. As the prominent metabolic disorder, hyperglycemia takes responsibilities

\section{Significance of this study}

What is already known about this subject?

- Endoplasmic reticulum stress protein kinase RNAlike endoplasmic reticulum kinase (PERK)/CEBP homologous protein (CHOP) pathway activation lead to tribbles homolog 3 (TRB3) upregulation.

- TRB3 participated in high glucose-induced insulin resistance in skeletal muscle.

- Nicorandil was known to decrease cytoplasmic calcium, oxidative stress and endoplasmic reticulum stress.

What are the new findings?

- Nicorandil attenuated high glucose-induced insulin resistance in skeletal muscle.

- Nicorandil suppressed PERK/CHOP pathwayinduced TRB3 upregulation by inhibiting oxidative stress-mediated endoplasmic reticulum stress.

How might these results change the focus of research or clinical practice?

- Nicorandil could be a promising drug in patients with ischemic heart disease complicated with diabetes.

for various complications of diabetes. A high glucose level is known as a factor inducing IR, which is termed as high glucose-induced IR. As one of the primary insulin-targeted tissues, skeletal muscle has been proved to dispose majority of glucose. The IR status of skeletal muscle is considered matching with the whole body IR. Glucose uptake impairment of skeletal muscle is a critical for high glucoseinduced IR.

Free glucose influx into skeletal muscle cells is dependent on the activation of glucose transport system. Insulin binds to insulin receptor and leads to phsophorylation of insulin receptor substrate 1 (IRS1), which further mediates the activation of phosphatidylinositol 3-kinase (PI3K)/Akt system. Akt phosphorylates its substrate Akt substrate 
$160 \mathrm{kDa}$ (AS160), which facilitates glucose transporter (GLUT) 4 translocation to plasma membrane. Activation of this IRS1/PI3K/Akt pathway was found impaired in IR. By physically occupying with the phosphorylation site of Akt, tribbles homolog (TRB) 3 was identified as a pseudokinase of Akt activity. ${ }^{1}$ Overexpression of TRB3 was found to impair skeletal muscle glucose uptake in high glucose- induced IR models. ${ }^{2}$

Excessive production of intracellular reactive oxygen species (ROS) is found in various cells types in diabetes mellitus. Mitochondria is believed as the major source of ROS, which is produced by mitochondrial glucose oxidation through nicotinamide adenine dinucleotide phosphate oxidases (NOX). Endoplasmic reticulum (ER) is an important organelle regulating multiple cellular functions such as lipid synthesis, calcium metabolism and protein maturation. When challenged by pathological stimuli such as oxidative stress, unfolded protein response is initiated to induce ER stress. According to our previous investigation, high glucose- induced ER stress was ROS-dependent. Consequently, activated ER stress protein kinase RNA-like endoplasmic reticulum kinase (PERK) signaling pathway led to expression of transcription factor $\mathrm{C} / \mathrm{EBP}$ homologous protein (CHOP). ${ }^{3}$ TRB3 was reported as an ER stress-inducible protein dependent on $\mathrm{CHOP}^{45}$

Nicorandil is now wildly used in treatment of ischemic heart diseases such as angina pectoris based on its pharmacological activity as a ATP-sensitive potassium $\left(\mathrm{K}_{\mathrm{ATP}}\right)$ opener, which could potently reduce cytoplasmic calcium content. Accumulating evidence have been proving nicorandil suppressed both oxidative stress and ER stress. Moreover, previous investigations also suggested nicorandil reduced activity of protein kinase $\mathrm{C}$ (PKC). The latter further activates NOX activity. Notably, the PKC activity was largely affected by cytoplasmic calcium level. Our pilot study suggested that nicorandil showed therapeutic effects on high glucose-induced IR. Thus, our interest was prompted to investigate the involved molecular mechanisms. We hypothesized that nicorandil could suppress ER stress PERK signaling-induced TRB3 expression by attenuating PKC/NOX system-mediated ROS production via reducing cytoplasmic calcium. Thus, nicorandil could increase the glucose uptake by maintaining Akt phosphorylation on insulin stimulation. We believe results from our current study would be beneficial in understanding the mechanisms of IR, and provide more evidence for new indications for nicorandil as a potential insulin sensitizer in the future.

\section{MATERIALS AND METHODS}

\section{Animal and treatments}

Adult Sprague-Dawley rats (aged 8 weeks, 280-300 g, non-littermates) were provided by Xi'an Jiaotong University Animal Experimental Center. Rats were maintained in independent polypropylene cages under controlled conditions (12-hour light-dark cycle; temperature
$25 \pm 1^{\circ} \mathrm{C}$; humidity $50 \%-60 \%$ ) and free to standard chow and sterilized water. In order to induce hyperglycemiainduced IR, animals received single intraperitoneal injection of streptozotocin (STZ, Sigma-Aldrich, dissolved in $10 \mathrm{mmol} / \mathrm{L}$ sodium citrate buffer, $\mathrm{pH}=4.5$ ) at dosage of $50 \mathrm{mg} / \mathrm{kg}$ bodyweight. Animals received vehicle buffer injections were considered as control. Two weeks after STZ injections, fasting blood glucose was detected with an automatic blood glucose analyzer (One Touch SureStep Meter, LifeScan). Four weeks after STZ injections, rats were administrated with nicorandil (Sigma-Aldrich) via drinking water at dosages of 5,10 and $15 \mathrm{mg} / \mathrm{kg} /$ day, respectively for 4 weeks. Thirty rats were used per treatment. Every 10 rats were used in subsequent glucose tolerance test (GTT), insulin tolerance test (ITT) and hyperinsulinemic-euglycemic clamp (HEC).

\section{Glucose tolerance test, insulin tolerance test}

The GTT and ITT were carried out according to the protocols described previously. ${ }^{2}$ Briefly, for GTT, animals were fasted overnight and then received intraperitoneal injection of glucose solution $(100 \mathrm{~g} / \mathrm{L})$ at dosage of $1 \mathrm{~g} /$ $\mathrm{kg}$ bodyweight. For ITT, fasted animals received intraperitoneal injection of insulin solution (Sigma-Aldrich) at dosage of $0.5 \mathrm{U} / \mathrm{kg}$ bodyweight. Blood samples were collected from tail vein before and 30, 60, $90120 \mathrm{~min}$ postglucose or insulin injections. Glucose concentrations were then determined. Moreover, based on the measurements, glucose concentration area under the curve, half-life time of glucose and glucose clearance rate were calculated.

\section{Hyperinsulinemic-euglycemic clamp}

HEC were performed according to several previous descriptions. ${ }^{6}$ Fasted animals were anesthetized with isoflurane inhalation (2\% for introducing and $4 \%$ for continuous anesthesia, $0.6 \mathrm{~L} / \mathrm{min}$ in oxygen). Femoral veins and left common carotid artery were exposed and catheterized. Catheters were flushed with heparinized saline. Specifically, blood samples were collected via left carotid artery; exogenous glucose and insulin infusions were accomplished via left and right femoral veins, respectively. After the blood samples were collected for baseline glucose concentration determination, insulin solution was infused at $10 \mathrm{mU} /(\mathrm{kg}$ bodyweight $\cdot \mathrm{min})$. At the same time, glucose solution $(100 \mathrm{~g} / \mathrm{L})$ was infused and its ratio was based on the 5 min glucose concentration. The infusion ratio was then adjusted to maintain blood glucose concentration constantly. Thus, within $90 \mathrm{~min}$, a stable ratio of exogenous glucose infusion rate (GIR) was acquired.

\section{Cell cullture and treatment}

L6 skeletal muscle cells (American Type Culture Collection) was maintained in Dulbecco's modified Eagle's medium (DMEM, Gibco) supplement with 15\% fetal bovine serum (Gibco), 2mmol/L L-glutamine (Sigma-Aldrich) and antibiotics mix (Sigma-Aldrich) in a humidified atmosphere 
composed of $5 \% \mathrm{CO}_{2}$ and $95 \%$ fresh air at $37^{\circ} \mathrm{C}$. L6 cells were cultured in medium containing $5 \mathrm{mmol} / \mathrm{L}$ glucose or $25 \mathrm{mmol} / \mathrm{L}$ glucose for $24 \mathrm{hours}$. Several cells were co-treated with medium containing nicorandil at final concentration of $100 \mu \mathrm{mol} / \mathrm{L}$.

\section{Cultured L6 cells glucose uptake evaluation}

The glucose uptake assay was carried out according to the protocols described before. ${ }^{7}$ Briefly, cells were deprived with serum-free DMEM incubation for 3 hours and treated with our without $100 \mathrm{nmol} / \mathrm{L}$ insulin for $45 \mathrm{~min}$. Cells were then incubated with Krebs buffer (containing $50 \mathrm{mmol} / \mathrm{L}$ HEPES (hydroxyethyl piperazineethanesulfonic acid), $4.7 \mathrm{mmol} / \mathrm{L} \mathrm{KCl}, 1.25 \mathrm{mmol} / \mathrm{L}$ $\mathrm{CaCl}_{2}, 1.25 \mathrm{mmol} / \mathrm{L} \mathrm{MgSO}_{4}, 136 \mathrm{mmol} / \mathrm{L} \mathrm{NaCl}, \mathrm{pH}=7.4$ )

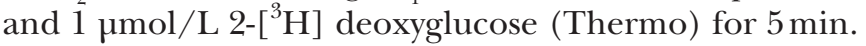
Pretreatment of $10 \mu \mathrm{mol} / \mathrm{L}$ cytochalasin B (SigmaAldrich) was used to determine the background glucose uptake. Then cells were washed with phosphate-buffered saline (PBS) and further lysed by $\mathrm{NaOH}$. Liquid scintillation counting was used to determine the radioactivity. The rate of glucose uptake was then calculated and normalized to protein levels which were determined by BCA (2,2-Biquinoline-4,4-dicarboxylic acid) assay.

\section{In situ ROS assessments}

The in situ ROS generation was assessed by dihydroethidium (DHE) staining as we described previously ${ }^{3} ; 10$ $\mu \mathrm{m}$ thick cryostat sections of skeletal muscle tissue were incubated with $10 \mu \mathrm{mol} / \mathrm{L} \mathrm{DHE} \mathrm{(Beyotime)} \mathrm{at} 37^{\circ} \mathrm{C}$ for 45 min in a humidified dark chamber. Cultured L6 cells were washed by PBS and then incubated with $20 \mu \mathrm{mol} / \mathrm{L}$ DHE in serum-free DMEM medium at $37^{\circ} \mathrm{C}$ for $30 \mathrm{~min}$ in a humidified dark chamber. Cell nuclei were stained with 2-(4-amidinophenyl)-6-indolecarbamidine dihydrochloride (DAPI, Invitrogen). A fluorescence microscopy was used to observe and capture the images that were further analyzed by Image J software.

\section{Oxidative stress status evaluations}

Total antioxidant capacity (TAC) assay and malondialdehyde (MDA) assessments were used to evaluate the oxidative stress status. Briefly, harvested tissue and cells were lysed by freeze-thaw cycle. Cell supernatants were resulted after centrifugation. A TAC assay kit (Beyotime) and a MDA assay kit (Beyotime) were used to detect TAC and MDA according to the instructions provided by the manufacturer.

\section{PKC and NOX activity determinations}

PKC and NOX activities in lysate of cultured L6 cells and skeletal muscle tissue were determined by a PKC kinase activity assay kit (Abcam) and a NOX kinase activity assay kit (Solarbio) according to the instructions provided by the manufacturers.

\section{Cytoplasmic free calcium $\left(\left[\mathrm{Ca}^{2+}\right]_{\mathrm{i}}\right)$ detection}

The detections were carried out according to our and others' previous descriptions with several modifications. ${ }^{8}$ Briefly, rats were anesthetized with isoflurane inhalation (2\% for introducing and $4 \%$ for continuous anesthesia, $0.6 \mathrm{~L} / \mathrm{min}$ in oxygen). Epitrochlearis muscles were exteriorized gently which were then superfused with Krebs-Henseleit buffer $(132 \mathrm{mmol} / \mathrm{L} \mathrm{NaCl}, 21.8 \mathrm{mmol} / \mathrm{L}$ $\mathrm{NaHCO}_{3}, 4.7 \mathrm{mmol} / \mathrm{L} \mathrm{KCl}, 2 \mathrm{mmol} / \mathrm{L} \mathrm{MgSO}, 2 \mathrm{mmol} / \mathrm{L}$ $\mathrm{CaCl}_{2}, \mathrm{pH}=7.4$ ) equilibrated with $95 \% \mathrm{~N}_{2}-5 \% \quad \mathrm{CO}_{2}$, in order to avoid tissue damage as much as possible. Skeletal muscles were loaded with Fura2/AM (Beyotime) for at final concentration of $5 \mathrm{mmol} / \mathrm{L}$ at $37^{\circ} \mathrm{C}$ for $30 \mathrm{~min}$ in dark. Electrodes were used to facilitate muscle contractions $(8$ $\mathrm{V}, 100 \mathrm{~Hz}, 3 \mathrm{~s}$ for 10 times). Then the muscle samples were embedded with optimal cutting temperature compound (Sakura) and made into $5 \mu \mathrm{m}$ cryostat sections in dark. L6 cells grow on cover glass were incubated with the calcium indicator Fura2/AM at final concentration of $10 \mu \mathrm{mol} / \mathrm{L}$ at $37^{\circ} \mathrm{C}$ in dark for $30 \mathrm{~min}$. After PBS washing, samples were observed with a fluorescence microscope. Fluorescent intensities were analyzed by ImageJ software and presented as $\left[\mathrm{Ca}^{2+}\right]_{\mathrm{i}}$ content in cytoplasm.

\section{Immunofluorescence staining}

The GLUT4 translocation behavior was observed by immunofluorescence staining. L6 cells grown on cover flips were fixed with $4 \%$ paraformaldehyde for $20 \mathrm{~min}$. After PBS washing, cells were treated with permeabilisation buffer ( $1 \%$ bovine serum albumin and $0.1 \%$ saponin) for $45 \mathrm{~min}$. Primary antibody against GLUT4 (Abcam, 1:200) was used to incubate the cells at $4^{\circ} \mathrm{C}$ overnight. After PBS washing, Alexa Fluor 488-conjugated secondary antibody (Abcam) was used to incubate the cells for $30 \mathrm{~min}$. DAPI was used to stain the nuclei. Cells were observed with an inverted fluorescence microscope.

\section{Subcellular fractionation}

The subcellular fractionations of cultured L6 cells were performed per the descriptions from a previousinvestigation. ${ }^{7}$ L6 cells were suspended in HES I buffer $(\mathrm{pH}=7.6)$ containing $0.25 \mathrm{mmol} / \mathrm{L}$ sucrose, $1 \mathrm{mmol} / \mathrm{L}$ EDTA, $20 \mathrm{mmol} / \mathrm{L}$ Tris and protease inhibitor cocktail (Abcam). Cells were homogenized and then centrifuged at $19000 \mathrm{~g}$ at $4^{\circ} \mathrm{C}$ for $20 \mathrm{~min}$. The resulted supernatant was further centrifuged at $175000 \mathrm{~g}$ for $75 \mathrm{~min}$ and then the pellets were considered as low-density microsomes fractions (LDM). The resulted pellets were layered on HES II buffer $(\mathrm{pH}=7.6)$ containing $1.12 \mathrm{mmol} / \mathrm{L}$ sucrose, $1 \mathrm{mmol} / \mathrm{L}$ EDTA and $20 \mathrm{mmol} / \mathrm{L}$ Tris. The pellets were then centrifuged at $100000 \mathrm{~g}$ at $4^{\circ} \mathrm{C}$ for $60 \mathrm{~min}$. The resulted pellets were considered as nuclear and mitochondrial fractions. Contents removed from the sucrose cushion were suspended into HES I buffer and further centrifuged at $41000 \mathrm{~g}$ at $4^{\circ} \mathrm{C}$ for $20 \mathrm{~min}$. The resulted pellets were considered as plasma membrane fractions (PM).

\section{Western blot analysis}

Cultured L6 cells and tissue homogenates were lysed with a lysis buffer system (Santa Cruz). The above subcellular fractionations were dissolved directly into the lysis buffer. Protein Extraction Kit (Beyotime) was used to extract the proteins. The concentrations of the proteins were determined by the 
BCA assay. Then the proteins were separated by sodium dodecyl sulfate polyacrylamide gel electrophoresis and then transferred to polyvinylidene difluoride membranes. After incubation with blocking buffer (Abcam), the membranes were further incubated with primary antibodies against glucose regulated protein (GRP)78 (Cell Signaling Tech, 1:2000), PERK (Cell Signaling Tech, 1:2000), phosphorylated PERK (p-PERK, Cell Signaling Tech, 1:2000), eukaryotic initiation factor (EIF) $2 \alpha$ (Abcam, 1:2500), phosphorylated EIF2 $\alpha$ (p-eIF2 $\alpha$, Abcam, 1:2500), activating transcription factor (ATF) 4 (Abcam, 1:2000), CHOP (Abcam, 1:2000), TRB3 (Abcam, 1:1000), Akt (Abcam, 1:2000), phosphorylated Akt (p-Akt, Abcam, 1:2000), AS160 (Abcam, 1:1000), phosphorylated AS160 (p-AS160, Abcam, 1:1000), GLUT4 (Cell Signaling Tech, 1:2000) and GAPDH (Abcam, $1: 4000)$ at $4^{\circ} \mathrm{C}$ for 10 hours. After TBST washing, membranes were incubated with HRP-conjugated secondary antibodies (Abcam) and then developed with SuperSignal West Pico PLUS Chemiluminescent substrate (Thermo). The immunoblots were visualized on X-ray films and further analyzed by Image J software.

\section{Statistics}

Data acquired in this study was presented in a $($ mean \pm SD) manner. Student's t-tests and one-way analysis of variance was used to analyze the differences between groups. NSK tests were carried out as post hoc tests. When $p$ value was
$<0.05$, the compared differences were considered statistically significant.

\section{RESULTS}

Nicorandil attenuated high glucose-induced IR in animals with diabetes

The animal study protocol is demonstrated in figure 1A. In order to study the impact of nicorandil on high glucose- induced IR, a hyperglycemia was induced by STZ peritoneal injection in rats. As demonstrated in figure 1D, significant hyperglycemia was detected 2 weeks after STZ injections and lasted during the whole experimental period. Nicorandil at dosages of 5, 10 and $15 \mathrm{mg} /$ $\mathrm{kg}$ was administrated to animals 4 weeks after STZ injection. Nicorandil showed no significant impacts on bodyweight at 4, 6 and 8 weeks and bodyweight area under the curve from 0 to 8 weeks after STZ injection (figure $1 \mathrm{~B}$ and C), fasting blood glucose levels (figure 1D) and glucose tolerance (figure $1 \mathrm{~F}$ ), glucose concentration area under the curve (online supplemental figure 1A), half-life time of glucose (online supplemental figure 1B) and glucose clearance rate (online supplemental figure 1C) in GTT. The glucose-induced IR was monitored by ITT and GIR. Significant IR could be observed 2 weeks after STZ injection and lasted to the end of experiment (figure 1G). Nicorandil administration significantly improved GIR and insulin tolerance in rats with diabetes (Figure 1E,G).
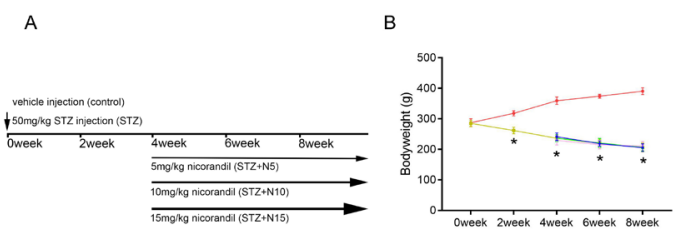

F
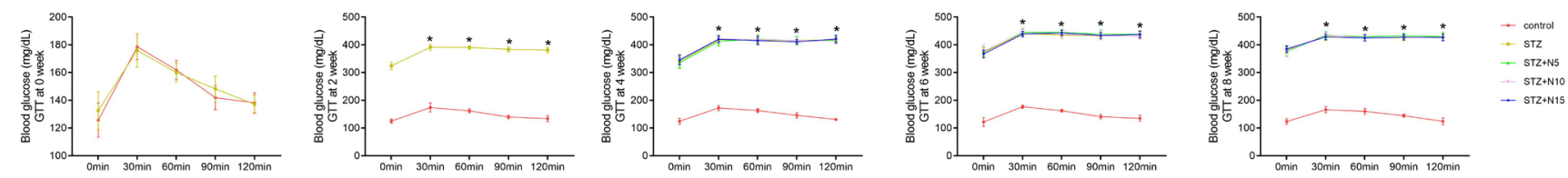

G
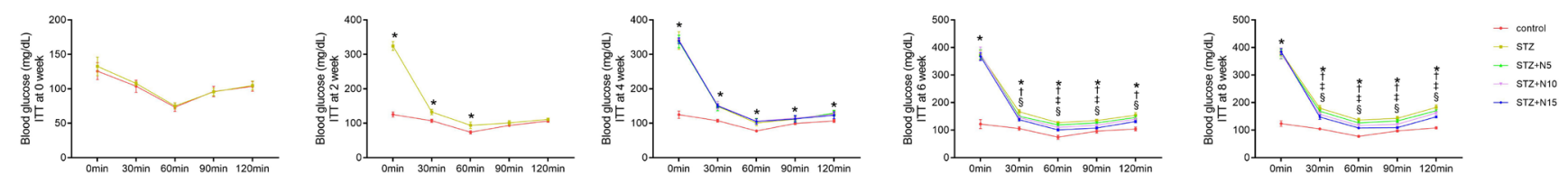

Figure 1 Effects of nicorandil on glucose metabolism in rats with diabetes. (A) The study protocol was plotted. Intraperitoneal streptozotocin (STZ) injection $(50 \mathrm{mg} / \mathrm{kg}$ ) was used to induce hyperglycemia of diabetes which was assigned as STZ group. Nicorandil at dosages of 5,10 and $15 \mathrm{mg} / \mathrm{kg}$ was administrated to animals 4 weeks after STZ injections which were assigned as STZ+N5, STZ+N10 and STZ+N15 groups, respectively. Bodyweight, fasting blood glucose (FBG), glucose infusion rate (GIR), glucose tolerance tests (GTT) and insulin tolerance tests (ITT) were monitored at 0, 2, 4, 6 and 8 weeks after STZ injections. Skeletal muscle tissues were harvested at the end of 8 weeks after STZ injection. (B) Bodyweights of animals were measured at 0, 2, 4, 6 and 8 weeks after STZ injections. (C) Calculated bodyweight area under the curve from 0 to 8 weeks. (D) Monitored FBG at 0, 2, 4, 6 and 8 weeks after STZ injections. (E) Measured GIR at 0, 2, 4, 6 and 8 weeks after STZ injections, which was used to evaluate whole body glucose uptake capacitiy. GTT and ITT were used to monitor glucose tolerance and insulin resistance in diabetic rats. (F) Results of GTT were plotted at 0, 2, 4, 6 and 8 weeks after STZ injections. (G) Results of ITT were plotted at 0, 2, 4, 6 and 8 weeks after STZ injections. Data were presented as (mean $\pm \mathrm{SD}$ ). ${ }^{\star} \mathrm{P}<0.05 \mathrm{vs}$ control; $\dagger \mathrm{p}<0.05 \mathrm{vs} \mathrm{STZ}$; $\neq \mathrm{p}<0.05$ vs STZ+N5; $\$ p<0.05$ vs STZ+N10 $(n=10)$. 

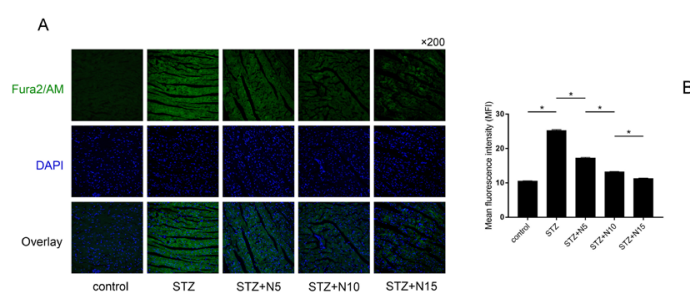

B
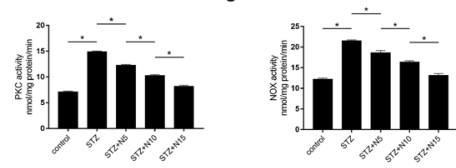

D
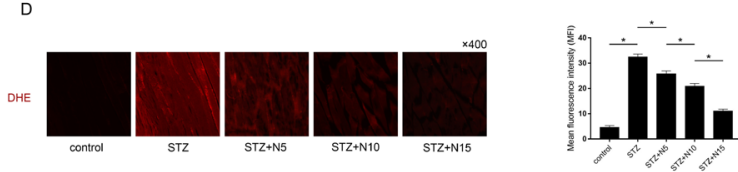

$E$
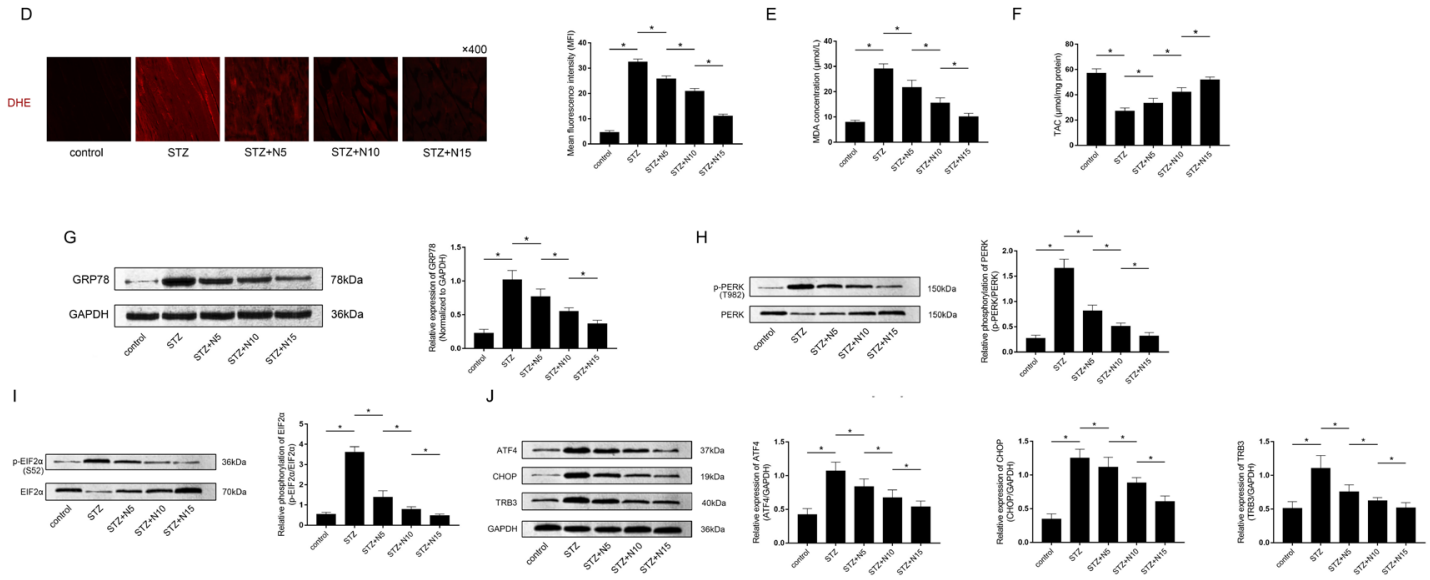

Figure 2 Effects of nicorandil on cytoplasmic free calcium, protein kinase C (PKC)/NAPDH oxidases (NOX) activities and oxidative stress-mediated protein kinase RNA-like endoplasmic reticulum kinase (PERK) signaling activation in skeletal muscle from IR rats with diabetes. (A) The calcium indicator Fura2/AM was used to stain the cytoplasmic free calcium ([Ca $\left.\left.{ }^{2+}\right]_{j}\right)$ on cryostat sections of skeletal muscle tissue. The captured fluorescent images were demonstrated. Columns indicated the detected mean fluorescent intensities of Fura2/AM staining of cryostat sections of skeletal muscle tissue harvested from control, STZ, STZ+N5, STZ+N10 and STZ+N15 groups, respectively. (B) and (C) Columns indicated the PKC and NOX activities of skeletal muscle tissue harvested from control, STZ, STZ+N5, STZ+N10 and STZ+N15 groups, respectively. (D) Generated reactive oxygen species (ROS) was stained with dihydroethidium (DHE) on cryostat sections of skeletal muscle tissue. Captured images of DHE staining of each group were demonstrated. Columns indicated mean fluorescent densities of DHE staining of cryostat sections of skeletal muscle tissue harvested from control, STZ, STZ+N5, STZ+N10 and STZ+N15 groups, respectively. (E) and (F) Columns indicated detected malondialdehyde (MDA) and total antioxidant capacity (TAC) of skeletal muscle tissue harvested from control, STZ, STZ+N5, STZ+N10 and STZ+N15 groups, respectively. (G) Immunoblots of GRP78 and GAPDH were demonstrated. Columns indicated the relative expression levels of GRP78, which is the hall marker of endoplasmic reticulum stress in skeletal muscle tissue harvested from control, STZ, STZ+N5, STZ+N10 and STZ+N15 groups, respectively. $(H)$ Immunoblots of phosphorylated PERK (p-PERK) and PERK were demonstrated. Columns indicated the relative phosphorylation levels of PERK in skeletal muscle tissue from control, STZ, STZ+N5, STZ+N10 and STZ+N15 groups, respectively. (I) Immunoblots of phosphorylated EIF2 $\alpha$ (p-EIF2 $\alpha$ ) and EIF2 $\alpha$ were demonstrated. Columns indicated the relative phosphorylation levels of EIF2 $\alpha$ in skeletal muscle tissue from control, STZ, STZ+N5, STZ+N10 and STZ+N15 groups, respectively. (J) Immunoblots of ATF4, CHOP, TRB3 and GAPDH were demonstrated. Columns indicated the relative expression levels of ATF4, CHOP, TRB3 and GAPDH in skeletal muscle tissue from control, STZ, STZ+N5, STZ+N10 and STZ+N15 groups, respectively. ${ }^{*} \mathrm{P}<0.05$ ( $\left.n=10\right)$. DAPI, 2-(4-amidinophenyl)-6-indolecarbamidine dihydrochloride; STZ, streptozotocin.

According to the ITT, nicorandil showed dramatic ameliorative effects on IR (figure 2B), glucose concentration area under the curve (online supplemental figure 2A), half-life time of glucose (online supplemental figure 2B) and glucose clearance rate (online supplemental figure 2C) at 6 and 8 weeks after STZ injection in a significant dose-dependent manner.

\section{Nicorandil alleviated $\left[\mathrm{Ca}^{2+}\right]$, accumulation and PKC/NOX system activities in skeletal muscle from IR rats with diabetes}

Skeletal muscle tissues were harvested 8 weeks after STZ injection. As demonstrated in figure 2A, nicorandil at dosages of 5,10 and $15 \mathrm{mg} / \mathrm{kg}$ significantly reduced $\left[\mathrm{Ca}^{2+}\right]_{\mathrm{i}}$ in diabetic IR skeletal muscle in a dosage-dependent manner. Moreover, the nicorandil administration also significantly decreased the
PKC and NOX activities in IR skeletal muscle in a dosagedependent manner (figure 2B and $\mathrm{C}$ ).

\section{Nicorandil attenuated oxidative stress-mediated ER stress in diabetic IR skeletal muscle}

Oxidative stress in skeletal muscle was assessed by DHE staining, MDA and TAC determinations. Specifically, intracellular ROS could be positively stained by DHE. MDA is the resulted product of oxidation of fatty acids in membrane which is also considered as an indicator for oxidative stress. TAC is the measurement of intracellular antioxidants content. As demonstrated in figure 2D, $\mathrm{E}$ and $\mathrm{F}$, nicorandil administration at 5, 10 and $15 \mathrm{mg} /$ $\mathrm{kg}$ dramatically reduced fluorescent intensities of DHE staining, MDA production and enhanced TAC in diabetic IR skeletal muscle in a dosage- dependent manner. As a 
hall marker of ER stress, expression of GRP78 was used to evaluate the degree of ER stress, which is demonstrated in figure 2G. The nicorandil treatments significantly downregulated relative expression levels of GRP78 in diabetic IR skeletal muscle tissue in a dosage-dependent manner.

\section{Nicorandil suppressed PERK/TRB3 pathway activation in skeletal muscle from IR rats with diabetes}

On ER stress, PERK signaling is activated by selfphosphorylation which further induces EIF2 $\alpha$ phosphorylation, causing upregulation of expressions of ATF4, CHOP and TRB3. Results in this study suggested that nicorandil administrated reduced phosphorylation levels of PERK and EIF2 $\alpha$ (figure $2 \mathrm{H}$ and $\mathrm{I}$ ) as well as expression levels of ATF4, CHOP and TRB3 (figure 2J) in skeletal muscle harvested from IR animals with diabetes in a dosage-dependent manner.

\section{Nicorandil reduced $\left[\mathrm{Ca}^{2+}\right]_{\text {accumulation and PKC/NOX system }}$ activities in high glucose-induced IR L6 cells}

Cultured L6 cells were exposed to high glucose incubation which was used to induce IR. Nicorandil at concentration of $100 \mu \mathrm{mol} / \mathrm{L}$ was used to co-treat the high glucose exposed L6 cells. Fura2/AM fluorescent staining was used to evaluate the $\left[\mathrm{Ca}^{2+}\right]_{i}$ accumulation in $\mathrm{L} 6$ cells. The results showed that nicorandil treatment significantly reduced fluorescent intensities of Fura2/AM (figure 3A) as well as PKC and NOX activities (figure 3B and $\mathrm{C}$ ) in high glucose incubated L6 cells.
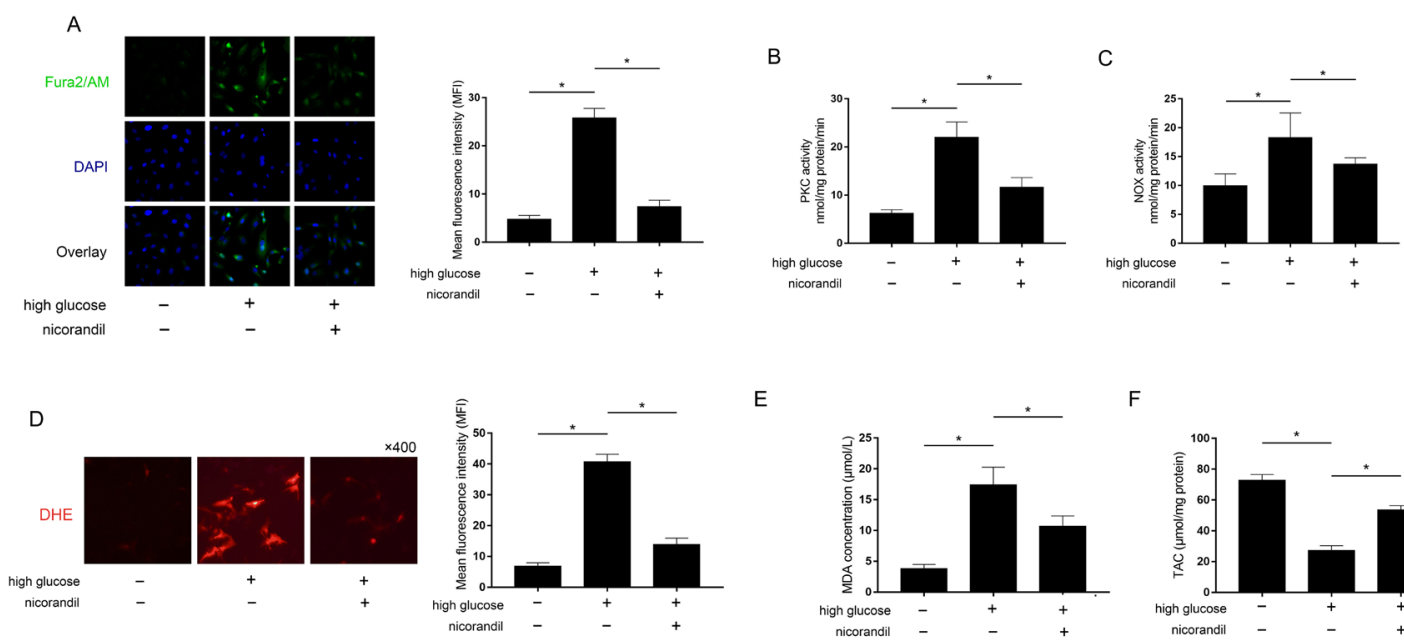

E
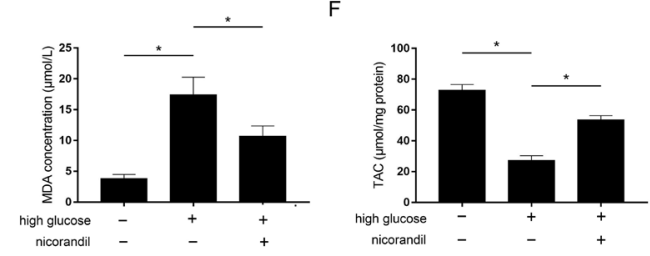

G
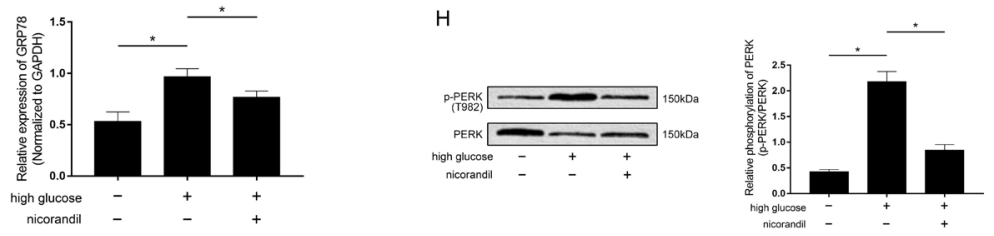
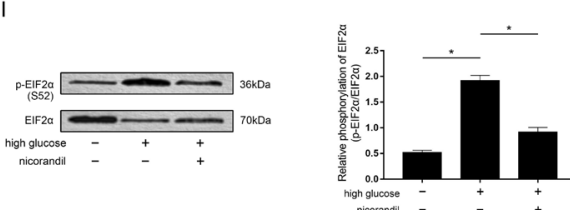

J

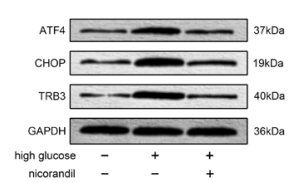

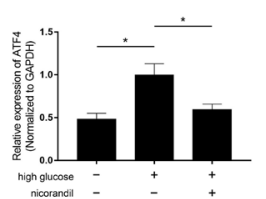

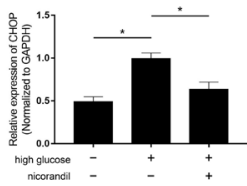

Figure 3 Effects of nicorandil on cytoplasmic free calcium, protein kinase C (PKC)/NAPDH oxidases (NOX) activities and oxidative stress-mediated protein kinase RNA-like endoplasmic reticulum kinase (PERK) signaling activation in high glucose incubated L6 muscle cells. (A) Fura2/AM staining was used to evaluate cytoplasmic free calcium ([Ca $\left.\left.{ }^{2+}\right]_{\mathrm{j}}\right)$ in cultured L6 cells. Captured fluorescent images of Fura2/AM, 2-(4-amidinophenyl)-6-indolecarbamidine dihydrochloride (DAPI) and their overlay were demonstrated. Columns indicated the mean fluorescent intensities of Fura2/AM in each group, respectively. (B) and (C) Columns indicated the PKC and NOX activities in L6 cells of each group, respectively. (D) Intracellular production of reactive oxygen species (ROS) was evaluated by dihydroethidium (DHE) staining. Captured fluorescent images of DHE staining of L6 cells were demonstrated. Columns indicated the mean fluorescent intensities of DHE staining in L6 cells from each group. (E) and $(F)$ Columns indicated the measured malondialdehyde (MDA) and total antioxidant capacity (TAC) in L6 cells from each group. (G) Immunoblots of GRP78 and GAPDH were demonstrated. Columns indicated the relative expression levels of GRP78 in $L 6$ cells from each group. $(\mathrm{H})$ Immunoblots of phosphorylated PERK ( $p$-PERK) and PERK were demonstrated. Columns indicated the relative phosphorylation levels of PERK in L6 cells from each group. (I) Immunoblots of phosphorylated EIF2 $\alpha$ $(p-E I F 2 \alpha)$ and EIF2 $\alpha$ were demonstrated. Columns indicated the relative phosphorylation levels of EIF2 $\alpha$ in L6 cells from each group. (J) Immunoblots of ATF4, CHOP, TRB3 and GAPDH were demonstrated. Columns indicated the relative expression levels of ATF4, CHOP, TRB3 and GAPDH in L6 cells from each group. ${ }^{*} P<0.05(n=10)$. 
Nicorandil attenuated oxidative stress-mediated ER stress in high glucose incubated L6 cells

DHE staining, MDA and TAC were used to evaluate oxidative stress in cultured L6 cells. High glucose incubation significantly triggered oxidative stress in cultured L6 cells. Nicorandil, however, dramatically decreased oxidative stress in high glucose exposed L6 cells, which was evidenced by decreased MFI of DHE staining (figure 3D), MDA (figure 3E) and increased TAC (figure 3F). Moreover, nicorandil treatment reduced expression levels of GRP78 which was considered as the hall marker of ER stress in high glucose incubated L6 cells (figure 3G).

\section{Nicorandil inhibited high glucose-induced PERK/TRB3} pathway activation in cultured L6 cells

The protein expression and phosphorylation levels in cultured L6 cells were evaluated by western blot analysis. The phosphorylation levels of PERK (figure $3 \mathrm{H}$ ) and EIF2 $\alpha$ (figure 3I) as well as the expression levels of ATF4, CHOP and TRB3 (figure 3J) were significantly upregulated in high glucose incubated L6 cells, which were dramatically reduced by nicorandil treatment.

Nicorandil facilitated insulin-induced Akt phosphorylation, GLUT4 translocation and glucose uptake in high glucose incubated L6 cells

The high glucose incubation dramatically impaired glucose uptake capability of L6 cells which could be considered as high glucose-induced IR (figure 4D). As shown in figure $4 \mathrm{~A}$, under circumstance of normal glucose incubation, insulin stimulation significantly induced Akt phosphorylation which further resulted in AS160 phosphorylation in L6 cells. The high glucose incubation, however, dramatically impaired insulin-stimulated Akt and AS160 phosphorylation. The nicorandil treatment was found effectively restored insulin-stimulated Akt and AS160 phosphorylation in high glucose incubated L6 cells. Western blot analysis detecting GLUT4 expression in different subcellular fractions was used to evaluate GLUT4 translocation. As demonstrated in figure 4B,

A
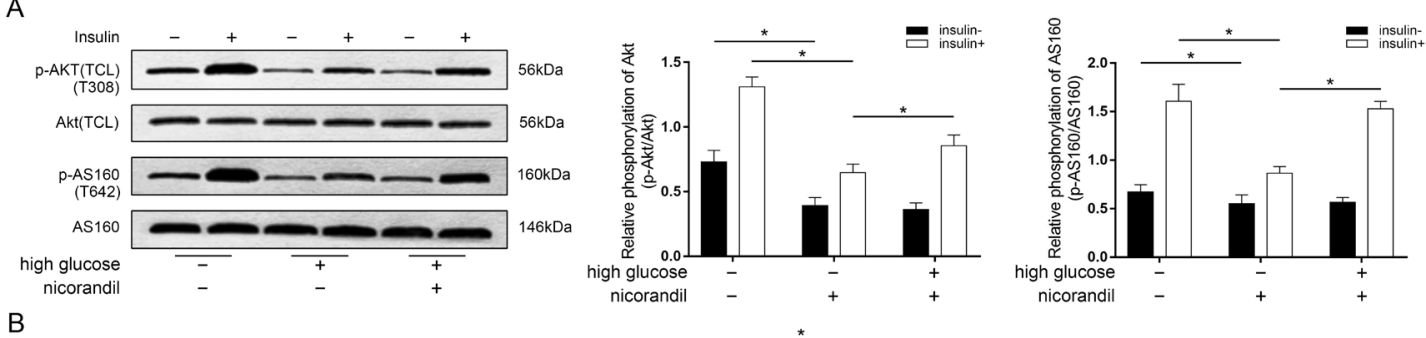

B

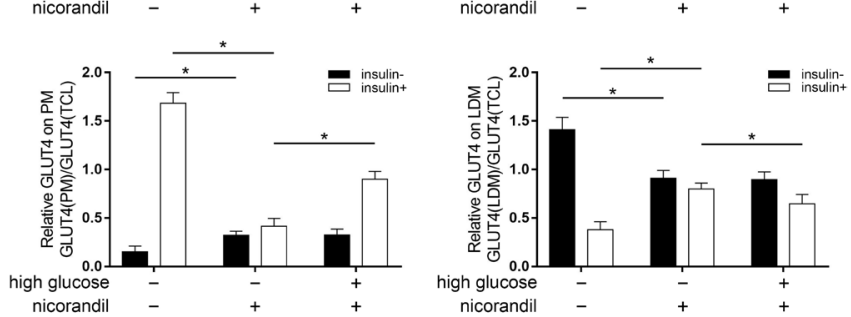

C

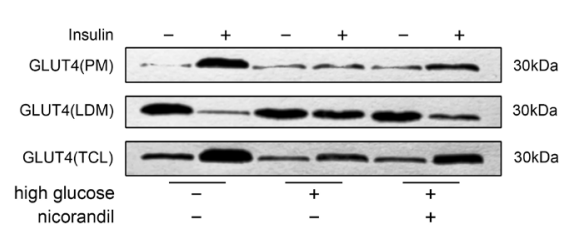
nicorandil

D
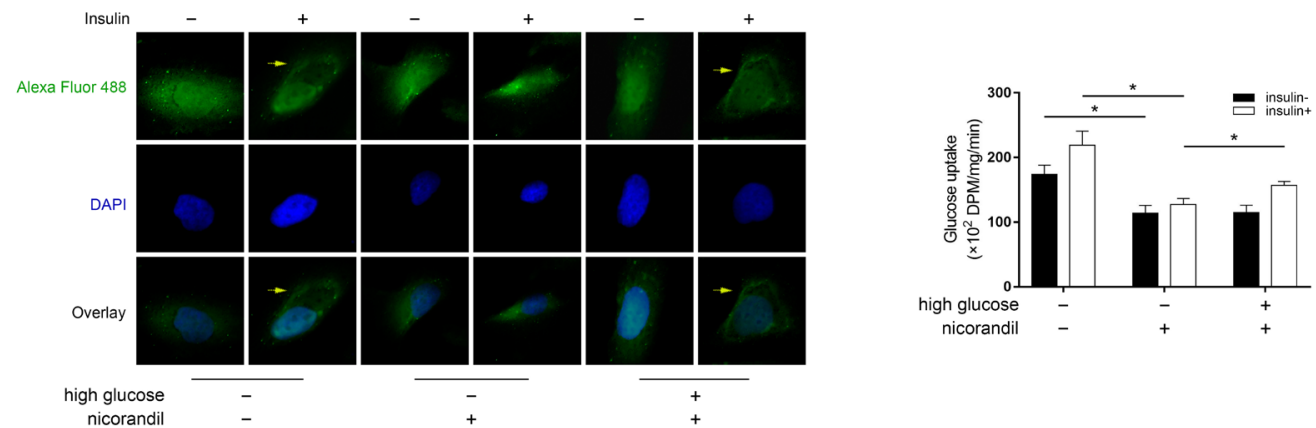

Figure 4 Effects of nicorandil on glucose uptake and Akt phosphorylation-mediated glucose transporter (GLUT)4 translocation in high-glucose incubated L6 cells. (A) Immunoblots of phosphorylated Akt (p-Akt), Akt, phosphorylated AS160 (p-AS160) and Akt substrate $160 \mathrm{kDa}$ (AS160). Columns indicated the relative phosphorylation levels of Akt and AS160 in L6 cells from each group received insulin stimulation (insulin+) and did not receive insulin stimulation (insulin-), respectively. (B) Immunoblots of GLUT4 in plasma membrane fractions (PM), low-density microsomes fractions (LDM) and total cell lysate (TCL) in L6 cells from each group. Columns indicated relative expression levels of GLUT4 on PM and LDM in L6 cells from each group received insulin stimulation (insulin+) and did not receive insulin stimulation (insulin-), respectively. (C) Representative fluorescent images of GLUT4 (stained by Alexa Fluor 488), 2-(4-amidinophenyl)-6-indolecarbamidine dihydrochloride (DAPI) and their overlay were demonstrated. Yellow arrows were pointing at accumulated Alexa Fluor 488 (indicating GLUT4) on PM. (D) Columns indicated the detected glucose uptake of cultured L6 cells from each group received insulin stimulation (insulin+) and did not receive insulin stimulation (insulin-), respectively. ${ }^{*} \mathrm{P}<0.05$. 


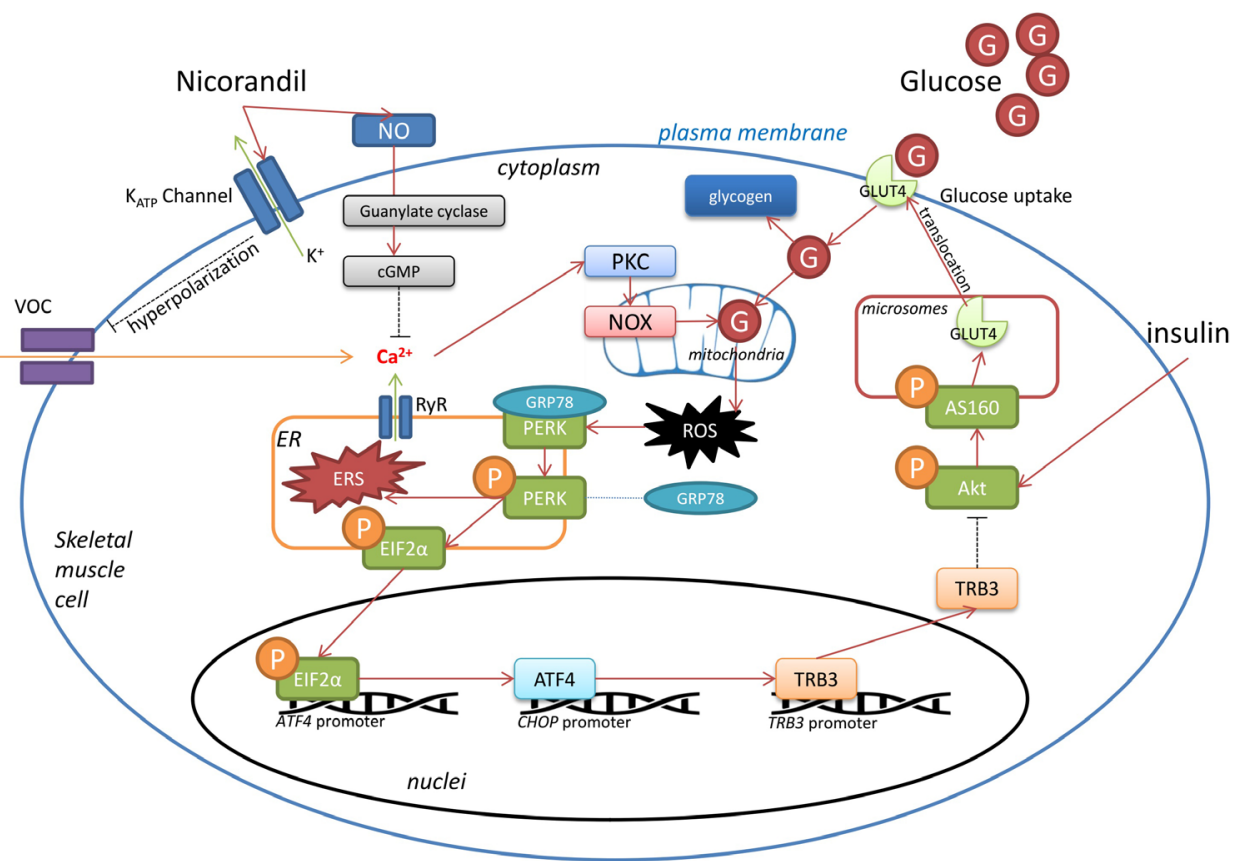

Figure 5 Schematic diagram demonstrating speculated mechanisms of nicorandil's activity on high glucose-induced insulin resistance. Under pathological condition of hyperglycemia, glucose is uptaken into skeletal muscle cells. Part of glucose was stored as glycogen. Redundant glucose experiences mitochondrial oxidation by nicotinamide adenine dinucleotide phosphate oxidases (NOX) and produces reactive oxygen species (ROS), which further mediates endoplasmic reticulum (ER) stress. As a sensor protein localized on ER membrane, protein kinase RNA-like endoplasmic reticulum kinase (PERK) is activated by selfphosphorylation up on ER stress. Phosphorylated PERK further phosphorylates EIF2 $\alpha$, which trigger transcription of ATF4. As a result, TRB3 expression is upregulated which is capable of inhibiting Akt phosphorylation. Akt phosphorylates its substrate AS160, which facilitates glucose transporter (GLUT)4 translocation to plasma membrane where glucose uptake takes place. Thus, the circumstance of hyperglycemia would abate the capability of glucose uptake in skeletal muscle cells, resulting in insulin resistance. Nicorandil was potent of reducing cytoplasmic free calcium by dual mechanisms. Nicorandil opens ATPsensitive potassium $\left(\mathrm{K}_{\text {ATP }}\right)$ channel, causing membrane hyperpolarizaiton, resulting in deactivation of VOC. Moreover, nicorandil facilities NO formation and thus decrease cytoplasmic free calcium via cGMP pathway. Decreased cytoplasmic free calcium would cause reduction of protein kinase C (PKC)/NOX activities, leading to alleviated ROS-mediated ER stress. Consequently, via PERK pathway described above, nocorandil mitigates hyperglycemia-induced insulin resistance in skeletal muscles. cGMP, cyclic guanosine monophosphate; NO, nitric oxide; VOC, voltage-operated channels.

GLUT4 expression was increased by nicorandil treatment in PM but decreased in LDM in high glucose incubated L6 cells. The representative fluorescent stating of GLUT4 in L6 cells were demonstrated in figure 4C. Under normal glucose condition, in response to insulin treatment, GLUT4 was found accumulated on plasma membrane area in L6 cells. Under high glucose condition, nevertheless, the accumulating behavior of GLUT4 on plasma membrane was found impaired which was recovered by nicorandil treatment. As a result, as demonstrated in figure $4 \mathrm{D}$, nicorandil treatment effectively restored the impaired glucose uptake in high- glucose incubated L6 cells.

\section{DISCUSSION}

Patients with prediabetes, type 1 diabetes mellitus and type 2 diabetes mellitus are all characterized by IR, which in turn contributes to the development of diabetes. Intensive insulin therapy and glycemic control are needed to reverse IR in patients with diabetes with sustained and poorly controlled hyperglycemia. ${ }^{10}$ There is a clinical scenario that hyperglycemia exacerbates IR, which is termed as glucose-induced IR. ${ }^{11}$ Several previous investigations suggested the association between $\mathrm{K}_{\text {ATP }}$ channels and IR. ${ }^{12}$ However, very few studies investigated the effects and underlying mechanisms of $\mathrm{K}_{\text {ATP }}$ openers in IR. In the present study, a $\mathrm{K}_{\text {ATP }}$ opener nicorandil was administrated to STZ-induced hyperglycemic IR rats. Established glucose tolerance impairment and hyperglycemia were not changed by nicorandil. However, evidenced by improved ITT and GIR, we found that nicorandil significantly attenuated whole body IR status. Skeletal muscle is a major organ disposing glucose. Malfunction of skeletal muscle glucose uptake is parallel to the whole body IR status. ${ }^{13}$ We made further investigations into the effects and underlying molecular mechanisms of nicorandil in alleviating high glucose-induced glucose uptake impairment in skeletal muscle in vivo and in vitro.

The anti-oxidant activity of nicorandil was well established in previous investigations. ${ }^{14}{ }^{15}$ Nicorandil is also considered as a ROS scavenger. ${ }^{16}$ Under circumstance of diabetes, hyperglycemia causes oxidative stress by glucose oxidation via NOX in mitochondria. ${ }^{17}$ PKC was reported to regulate NADPH oxidases activity by 
mediating activation of small GTP binding to Rac1, which activates $\mathrm{p} 47^{\text {phox }}{ }^{18}$ It is believed that $\left[\mathrm{Ca}^{2+}\right]_{\mathrm{i}}$ elevation facilitated the association and interaction between PKC and DAG within membranes which stimulated PKC enzymatic activity. ${ }^{19}$ Notably, many previous studies have showed that nicorandil was potent in lowering $\left[\mathrm{Ca}^{2+}\right]_{\mathrm{i}}$ in vascular smooth muscle cells and myocytes. ${ }^{20}{ }^{21}$ In the present study, we used fluorescent intensity of calcium indicator Fura2/AM to assess $\left[\mathrm{Ca}^{2+}\right]_{\mathrm{i}}$ in skeletal muscle cells at contractive state, which was similar with several previous investigations. ${ }^{22}{ }^{23}$ It would more accurate if absorption spectrophotometry was used to quantitate $\left[\mathrm{Ca}^{2+}\right]_{\mathrm{i}}$. We found nicorandil reduced $\left[\mathrm{Ca}^{2+}\right]_{\mathrm{i},}$ and consequently lowered PKC and NOX activities in both diabetic IR skeletal muscle tissue and high glucose exposed IR skeletal muscle cells. Eventually, nicorandil suppressed oxidative stress, which was in accordance with the results from previous investigations. ${ }^{24}$ Here, we reported a novel mechanism that nicorandil could attenuate intracellular oxidative stress via $\left[\mathrm{Ca}^{2+}\right]_{\mathrm{i}} / \mathrm{PKC} / \mathrm{NOX}$ pathway.

ER is an important and specialized organelle involved in many fundamental cell functions. ER stress is triggered when the cells were challenged by oxidative stress, a paradigm termed as oxidative stress-mediated ER stress. ${ }^{25}$ As a sensor protein localized on ER membrane, PERKmediated pathway was activated in this case, which was described by our previous study. ${ }^{3}$ Phosphorylated PERK further phosphorylates the eIF2 $\alpha$ which takes charge of shutting down global gene transcription but initiates expression of acute phase genes such as ATF4. The transcription factor CHOP, whose expression is strongly dependent on ATF4, was proved to initiate TRB3 expression by binding to the amino acid response elements of the tunicamycin response region of $t r b 3$ promoter. $^{4}$ Previous investigations have identified TRB3 as a pathogenic factor causing glucose-induced IR in skeletal muscle. ${ }^{26}$ Indicated by GRP78 expression and PERK phosphorylation alterations, results from the current study suggested nicorandil inhibited the activation of ER stress PERK signaling pathway. As a result, the expression levels of ATF4, CHOP and TRB3 were also reduced. Thus, we could propose that nicorandil may alleviate high glucose-induced IR by blocking PERK/ eIF2 $\alpha$ / ATF4/CHOP/TRB3 signaling transduction in skeletal muscle.

It is accepted that insulin-stimulated cellular glucose transportation is fundamental to tissue glucose uptake capability, which decides the severity of IR. In this study, we found that nicorandil significantly restored insulinstimulated glucose uptake in cultured skeletal muscle cells exposed to high glucose incubation. Akt phosphorylation plays a critical role in mediating glucose uptake by phosphorylating its substrate AS160 to facilitate GLUT4 traffic from intracellular compartments such as microsomes to plasma membrane to ingest glucose. ${ }^{727}$ According to previous reports, overexpression of TRB3 worsened IR by impairing skeletal muscle glucose uptake capability. ${ }^{26}$ The impact of TRB3 on IR is largely dependent on its role of blocking Akt phosphorylation in response to insulin by direct binding. ${ }^{1}$ In this study, we found that nicorandil treatment dramatically increased Akt phosphorylation in response to insulin stimulation in high-glucose exposed skeletal muscle cells because nicorandil downregulated TRB3 expression. Consequently, nicorandil treatment upregulated AS160 phosphorylation and GLUT4 plasma membrane translocation.

Thus, a relatively clear molecular mechanism for nicorandil in attenuating high glucose-induced IR could be plotted, which is demonstrated in figure 5 . By activating $\mathrm{K}_{\mathrm{ATP}}$, nicorandil decreased cytoplasmic calcium concentration, leading to reduction of enzymatic activities of PKC/NOX system. Thus, less ROS is generated by glucose oxidation with NOX. Oxidative stress-mediated ER stress PERK/eIF2 $\alpha$ / ATF4/CHOP signaling activation is alleviated by nicorandil. As a result, expression of TRB3 is downregulated in skeletal muscle cells. Thus, on insulin stimulation, Akt phosphorylation could take place more readily. With the aid of phosphorylated AS160, more GLUT4 would accomplish plasma membrane translocation to enhance cellular glucose uptake capability, resulting in IR improvement. Data collected in the present study proposed molecular mechanism underlying a novel pharmacological activity of nicorandil in alleviating high glucose-induced IR. Along with its cardioprotective effects, nicorandil could be deemed as a potential optimized agent in treatment of patients with ischemic heart diseases complicated with diabetes.

Contributors ZL, PL and YZ designed the study, performed experiments, analyzed the data and wrote the manuscript. $\mathrm{HZ}$ accomplished the statistics. CH, TH, SP, LZ, NZ and GG participated in the experiments. JW reviewed, revised and edited the manuscript.

Funding This study was supported by Innovative Talents Promotion Project of Shaanxi Province (2019KJXX-019); Fundamental Scientific Research Foundation of Xi'an Jiaotong University (xzy012019131); Health Scientific Foundation of Shaanxi Province (2018E11); Natural Science Basic Research Foundation of Shaanxi Province (2020JQ-941).

Competing interests None declared.

Patient consent for publication Not required.

Ethics approval The experimental protocols were reviewed and approved by Institutional Animal Care and Use Committee of Medical Research Institute of Northwestern Polytechnical University.

Provenance and peer review Not commissioned; externally peer reviewed.

Data availability statement Data are available on reasonable request.

Supplemental material This content has been supplied by the author(s). It has not been vetted by BMJ Publishing Group Limited (BMJ) and may not have been peer-reviewed. Any opinions or recommendations discussed are solely those of the author(s) and are not endorsed by BMJ. BMJ disclaims all liability and responsibility arising from any reliance placed on the content. Where the content includes any translated material, BMJ does not warrant the accuracy and reliability of the translations (including but not limited to local regulations, clinical guidelines, terminology, drug names and drug dosages), and is not responsible for any error and/or omissions arising from translation and adaptation or otherwise.

Open access This is an open access article distributed in accordance with the Creative Commons Attribution Non Commercial (CC BY-NC 4.0) license, which permits others to distribute, remix, adapt, build upon this work non-commercially, and license their derivative works on different terms, provided the original work is properly cited, appropriate credit is given, any changes made indicated, and the use is non-commercial. See: http://creativecommons.org/licenses/by-nc/4.0/. 
ORCID ID

Junkui Wang http://orcid.org/0000-0002-4478-5335

\section{REFERENCES}

1 Du K, Herzig S, Kulkarni RN, et al. Trb3: a tribbles homolog that inhibits Akt/PKB activation by insulin in liver. Science 2003;300:1574-7.

2 Zhang W, Wu M, Kim T, et al. Skeletal muscle TRIB3 mediates glucose toxicity in diabetes and high- fat diet-induced insulin resistance. Diabetes 2016;65:2380-91.

3 Liu Z-W, Zhu H-T, Chen K-L, et al. Protein kinase RNA-like endoplasmic reticulum kinase (PERK) signaling pathway plays a major role in reactive oxygen species (ROS)-mediated endoplasmic reticulum stress-induced apoptosis in diabetic cardiomyopathy. Cardiovasc Diabetol 2013;12:158.

4 Ohoka N, Yoshii S, Hattori T, et al. Trb3, a novel ER stress-inducible gene, is induced via ATF4-CHOP pathway and is involved in cell death. Embo J 2005:24:1243-55.

5 Tsai D-H, Chung C-H, Lee K-T. Antrodia cinnamomea induces autophagic cell death via the CHOP/TRB3/Akt/mTOR pathway in colorectal cancer cells. Sci Rep 2018;8:17424.

6 Morris EM, Meers GME, Ruegsegger GN, et al. Intrinsic high aerobic capacity in male rats protects against diet-induced insulin resistance. Endocrinology 2019;160:1179-92.

7 Sun Y, Côté J-F, Du K. Elmo2 is a regulator of insulin-dependent GLUT4 membrane translocation. J Biol Chem 2016;291:16150-61.

8 Liu Z, Zhang Y, Qiu C, et al. Diabetes mellitus exacerbates postmyocardial infarction heart failure by reducing sarcolipin promoter methylation. ESC Heart Fail 2020;7:1935-48.

9 Arias EB, Kim J, Cartee GD. Prolonged incubation in PUGNAC results in increased protein $\mathrm{O}$-linked glycosylation and insulin resistance in rat skeletal muscle. Diabetes 2004;53:921-30.

10 Unger RH, Grundy S. Hyperglycaemia as an inducer as well as a consequence of impaired islet cell function and insulin resistance: implications for the management of diabetes. Diabetologia 1985;28:119-21.

11 Zhang H-H, Ma X-J, Wu L-N, et al. Sirt1 attenuates high glucoseinduced insulin resistance via reducing mitochondrial dysfunction in skeletal muscle cells. Exp Biol Med 2015;240:557-65.

12 Wasada T. Adenosine triphosphate-sensitive potassium (K(ATP)) channel activity is coupled with insulin resistance in obesity and type 2 diabetes mellitus. Intern Med 2002;41:84-90.

13 Abdul-Ghani MA, DeFronzo RA. Pathogenesis of insulin resistance in skeletal muscle. J Biomed Biotechnol 2010;2010:1-19.
14 Asensio-López MC, Soler F, Pascual-Figal D, et al. DoxorubicinInduced oxidative stress: the protective effect of nicorandil on $\mathrm{HL}-1$ cardiomyocytes. PLoS One 2017;12:e0172803.

15 Ravindran S, Swaminathan K, Ramesh A, et al. Nicorandil attenuates neuronal mitochondrial dysfunction and oxidative stress associated with murine model of vascular calcification. Acta Neurobiol Exp 2017;77:57-67

16 Kasono K, Yasu T, Kakehashi A, et al. Nicorandil improves diabetes and rat islet beta-cell damage induced by streptozotocin in vivo and in vitro. Eur J Endocrinol 2004;151:277-85.

17 Leto TL, Morand S, Hurt D, et al. Targeting and regulation of reactive oxygen species generation by Nox family NADPH oxidases. Antioxid Redox Signal 2009;11:2607-19.

18 Remijsen QFM, Fontayne A, Verdonck F, et al. The antimicrobial peptide parabutoporin competes with p47(phox) as a PKC-substrate and inhibits NADPH oxidase in human neutrophils. FEBS Lett 2006;580:6206-10.

19 Huang KP. The mechanism of protein kinase $\mathrm{C}$ activation. Trends Neurosci 1989;12:425-32.

$20 \mathrm{Kai} \mathrm{H}$, Kanaide H, Nakamura M. Effects of nicorandil on cytosolic calcium concentrations in quin2-loaded rat aortic vascular smooth muscle cells in primary culture. J Pharmacol Exp Ther 1989;251:1174-80.

21 Wei J, Watanabe Y, Takeuchi K, et al. Nicorandil stimulates a $\mathrm{Na}^{+}$/ $\mathrm{Ca}^{2+}$ exchanger by activating guanylate cyclase in guinea pig cardiac myocytes. Pflugers Arch 2016;468:693-703.

22 Eshima H, Miura S, Senoo N, et al. Improved skeletal muscle $\mathrm{Ca}^{2+}$ regulation in vivo following contractions in mice overexpressing PGC-1 $\alpha$. Am J Physiol Regul Integr Comp Physiol 2017;312:R1017-28

23 Sonobe T, Inagaki T, Poole DC, et al. Intracellular calcium accumulation following eccentric contractions in rat skeletal muscle in vivo: role of stretch-activated channels. Am J Physiol Regul Integr Comp Physiol 2008;294:R1329-37.

24 Serizawa K-I, Yogo K, Aizawa K, et al. Paclitaxel-Induced endothelial dysfunction in living rats is prevented by nicorandil via reduction of oxidative stress. J Pharmacol Sci 2012;119:349-58.

25 Ding W, Yang L, Zhang M, et al. Reactive oxygen species-mediated endoplasmic reticulum stress contributes to aldosterone-induced apoptosis in tubular epithelial cells. Biochem Biophys Res Commun 2012;418:451-6.

26 Zhang W, Liu J, Tian L, et al. Trib3 mediates glucose-induced insulin resistance via a mechanism that requires the hexosamine biosynthetic pathway. Diabetes 2013;62:4192-200.

27 Gallo MP, Femminò S, Antoniotti S, et al. Catestatin induces glucose uptake and GLUT4 trafficking in adult rat cardiomyocytes. Biomed Res Int 2018;2018:1-7. 